\title{
COUNTEREXAMPLES TO THE UNIQUE AND COFINAL BRANCHES HYPOTHESES
}

ITAY NEEMAN AND JOHN STEEL

\begin{abstract}
We produce counterexamples to the unique and cofinal branches hypotheses, assuming (slightly less than) the existence of a cardinal which is strong past a Woodin cardinal.
\end{abstract}

Martin-Steel [3] introduced the notion of an iteration tree, and with it the question of iterability: the existence of a method for choosing cofinal branches through iteration trees in a manner that secures the wellfoundedness of the models generated by the trees. The question has since become central to the study of large cardinals, with particular importance to the construction of inner models and to proofs of determinacy. (For specific examples see Neeman [4] and Neeman [5].)

Martin-Steel [3] suggested a natural hypothesis, which if true, would combine with the results they proved to yield iterability for countable elementary substructures of rank initial segments of $\mathrm{V}$, enough for the applications to proofs of determinacy, and some simple applications to inner model theory. (In the deeper parts of inner model theory, one wants to construct iterable models satisfying large cardinal hypotheses in situations in which there may be no large cardinals in V. Here the possibly vacuous iterability of elementary submodels of $\mathrm{V}$ is irrelevant.) The hypothesis, known as the Unique Branches Hypothesis, or UBH for short, states that every countable iteration tree on $\mathrm{V}$ has at most one cofinal wellfounded branch. Combined with the results of Martin-Steel [3] it would imply that in fact every countable iteration tree on $\mathrm{V}$ which is sufficiently closed has exactly one cofinal wellfounded branch.

Martin-Steel [3] also coined the Cofinal Branches Hypothesis, CBH for short, stating that every countable iteration tree on $\mathrm{V}$ has at least one cofinal wellfounded branch. For sufficiently closed iteration trees this is a consequence of UBH.

Woodin refuted UBH about two years after it had been introduced, using very large cardinals involving embeddings of a rank initial segment of the universe into itself. In 1999, Woodin went on to refute CBH under the weaker hypothesis that there is a supercompact cardinal with a Woodin cardinal above it; this gave a counterexample to UBH under the same hypothesis. The counterexample to UBH, which Woodin presented at the meeting on Core Model Theory at the American Institute of Mathematics in December 2004, has a very simple

This material is based upon work supported by the National Science Foundation under Grants No. DMS-0094174 (Neeman) and DMS-0401312 (Steel). 
tree order, involving a single ultrapower by an extender $F$ followed by an alternating chain on the ultrapower model. The tree order associated to Woodin's counterexample to $\mathrm{CBH}$ is also very simple, involving a single ultrapower by an extender $F$ followed by a composition of $\omega$ alternating chains on the ultrapower model.

In this paper we construct counterexamples to $\mathrm{UBH}$ and $\mathrm{CBH}$ using a cardinal substantially smaller than supercompact. The precise large cardinal assumption we use is stated in conditions (A1) and (A2) in Section 1. It is slightly weaker than the assumption that there is an extender which is strong past a Woodin cardinal.

Our counterexamples have the same simple tree structures used by Woodin. The structure of the counterexample to UBH, involving a single ultrapower followed by an alternating chain, is illustrated in Diagram 1. The structure of the counterexample to $\mathrm{CBH}$, involving a single ultrapower followed by $\omega$ alternating chains, is illustrated in Diagram 2.

In both our counterexamples the first extender used is overlapped. (An iteration tree $\mathcal{U}$ has an overlap at $\xi$ if $\operatorname{crit}\left(F_{\xi}\right)<\operatorname{spt}\left(F_{\alpha}\right)$ for some $\alpha<\xi$ so that $\alpha+1 U \xi+1$, and $F_{\alpha}$ is said to be overlapped in such a situation. $\mathcal{U}$ is non-overlapping if it has no overlaps.) It should also be noted that the first extender used, in both counterexamples, is not countably closed.

Our counterexample to UBH shows clearly why UBH ought to have been restricted to non-overlapping iteration trees. Let $F$ be the first extender used in our counterexample to $\mathrm{UBH}$, and $\mathcal{T}$ be the alternating chain on $\mathrm{Ult}(\mathrm{V}, F)$ which constitutes the remainder of the counterexample. Because of the overlap $\mathcal{T}$ can also be regarded as a tree on $\mathrm{V}$. So regarded, $\mathcal{T}$ has exactly one wellfounded branch. However, if $\mathcal{T}$ is regarded as a tree on the weaker model Ult $(\mathrm{V}, F)$, as it may be if overlapping is allowed, then both its branch models are wellfounded, and in fact more than that, they are equal! Thus our counterexample relies heavily on the fact that in an overlapping tree, one is allowed to discard information which might differentiate cofinal branches at limit stages.

Our method will not produce a countably closed counterexample to UBH for a similar reason: if $\operatorname{Ult}(\mathrm{V}, F)$ is countably closed, then it is too close to $\mathrm{V}$ to hide the distinction between the branches of $\mathcal{T}$. So far as we can see, overlapping does not help produce a counterexample in the countably closed case.

Woodin's counterexamples involve non-overlapping iteration trees. They are not countably closed, and indeed it is not known if there is any counterexample to UBH or to $\mathrm{CBH}$ in which all extenders used are countably closed in the models in which they appear. The question is important since the restriction of UBH to non-overlapping iteration trees which only use countably closed extenders would suffice for the existing applications of UBH.

The main results on UBH in the positive direction are those of [3], [7], and [6]. The paper [3] shows that if there is any counterexample to $\mathrm{UBH}$, then there is a $\delta$ which is Woodin in $L\left(\mathrm{~V}_{\delta} \cup\left\{\mathrm{V}_{\delta}^{\sharp}\right\}\right)$. The paper [7] shows that if there is a nonoverlapping counterexample to UBH, then there is an iterable inner model with infinitely many Woodin cardinals. Finally, [6] shows that in any iterable $L[\vec{E}]$ model, UBH holds for non-overlapping "plus two" iteration trees using extenders 
from the images of the coherent sequence. (The plus two condition there is a stronger condition than the one we define below.)

$\S 1$. Preliminaries. We work throughout the paper under the assumption that there exists a cardinal $\delta$ and an extender $F$ so that:

(A1) $\operatorname{crit}(F)<\delta, \operatorname{spt}(F)=\delta$, and $F$ is $\delta$-strong (meaning that $\mathrm{V}_{\delta} \subset \mathrm{Ult}(\mathrm{V}, F)$ ). (A2) $\delta$ is Woodin in the smallest admissible set containing $\mathrm{V}_{\delta} \cup\{F\}$.

Let $\delta$ and $F$ witness conditions (A1) and (A2), with $\delta$ least possible. The following lemma shows that $\delta$ must have cofinality $\omega$.

Lemma 1.1. Let $\delta^{*}$ be a limit cardinal of cofinality $>\omega$ and let $A^{*} \subset \mathrm{V}_{\delta^{*}}$. Let $\varphi\left(v_{1}, v_{2}\right)$ be a $\Pi_{1}$ formula. Let $K^{*}$ be the smallest admissible set containing $\mathrm{V}_{\delta^{*}} \cup\left\{\delta^{*}, A^{*}\right\}$. Suppose that $\delta^{*}$ is inaccessible in $K^{*}$ and that $K^{*} \models \varphi\left[\delta^{*}, A^{*}\right]$. Then there is $\bar{\delta}<\delta^{*}$ so that $\bar{K} \models \varphi[\bar{\delta}, \bar{A}]$ where $\bar{A}=A^{*} \cap \mathrm{V}_{\bar{\delta}}$ and $\bar{K}$ is the smallest admissible set containing $V_{\bar{\delta}} \cup\{\bar{\delta}, \bar{A}\}$.

Lemma 1.1 cannot be proved simply by constructing a $\Sigma_{1}$ elementary substructure of $K^{*}$, since admissibility is not characterized by a $\Pi_{2}$ sentence.

Proof of Lemma 1.1. Working in $K^{*}$ let $R$ be the tree of attempts to construct a sequence $\left\langle\delta_{n}, K_{n}, a_{n}, \pi_{n} \mid n<\omega\right\rangle$ so that:

1. $\delta_{n}<\delta^{*}, \delta_{n}$ is a strong limit cardinal, $K_{n} \in \mathrm{V}_{\delta^{*}}$, and $K_{n}$ is transitive.

2. $\mathrm{V}_{\delta_{n}} \cup\left\{\delta_{n}, A^{*} \cap \mathrm{V}_{\delta_{n}}\right\} \subset K_{n}$, and $K_{n} \models \varphi\left[\delta_{n}, A^{*} \cap \mathrm{V}_{\delta_{n}}\right]$.

3. $\pi_{n}: K_{n} \rightarrow K_{n+1}$ is elementary, $\operatorname{crit}\left(\pi_{n}\right)=\delta_{n}, \pi_{n}\left(\delta_{n}\right)=\delta_{n+1}$, and $\pi_{n}\left(A^{*} \cap\right.$ $\left.\mathrm{V}_{\delta_{n}}\right)=A^{*} \cap \mathrm{V}_{\delta_{n+1}}$.

4. $a_{n} \in K_{n}$ is an ordinal, and $a_{n+1}<\pi_{n}\left(a_{n}\right)$.

First we show that $R$ is illfounded. Suppose for contradiction that $R$ is wellfounded and let $\alpha$ be its rank. Since $R$ belongs to $K^{*}$, and since $K^{*}$ is admissible, $\alpha$ belongs to $K^{*}$. Let $K^{*} \| \alpha$ denote $\mathrm{L}_{\omega \cdot \alpha}\left(\mathrm{V}_{\delta^{*}} \cup\left\{A^{*}\right\}\right)$. For each node $p \in R$ let $\rho(p)$ be the rank of $p$ in $R$. Thus $\rho: R \rightarrow \alpha$ is order preserving and onto. Let $\alpha_{0}=\rho(\emptyset)<\alpha$. Working by induction construct a sequence $\left\langle H_{n}, \alpha_{n+1} \mid n<\omega\right\rangle$ subject to conditions (a) $-(\mathrm{c})$ :

(a) $H_{n} \in K^{*}$ is an elementary substructure of $K^{*} \| \alpha,\left\{\alpha_{n}, \delta^{*}, \mathrm{~V}_{\delta^{*}}, A^{*}\right\} \subset H_{n}$, and $H_{n} \cap \mathrm{V}_{\delta^{*}}=\mathrm{V}_{\delta_{n}}$ for some $\delta_{n}<\delta^{*}$.

(b) $H_{n-1} \prec H_{n}$ if $n>0$ (so that id: $H_{n-1} \rightarrow H_{n}$ is elementary).

Let $K_{n}$ be the transitive collapse of $H_{n}$ and let $\sigma_{n}: K_{n} \rightarrow H_{n}$ be the anticollapse embedding. Let $a_{n}=\sigma_{n}{ }^{-1}\left(\alpha_{n}\right)$. If $n>0$ then let $\pi_{n-1}: K_{n-1} \rightarrow K_{n}$ be the embedding ${\sigma_{n}}^{-1} \circ$ id $\circ \sigma_{n-1}$.

(c) $\alpha_{n+1}$ is equal to $\rho\left(\left\langle\delta_{i}, K_{i}, a_{i}, \pi_{i} \mid i \leq n\right\rangle\right)$.

It is easy to verify inductively that (i) $\left\langle\delta_{i}, K_{i}, a_{i}, \pi_{i} \mid i \leq n\right\rangle$ is a node in $R$; and (ii) $\alpha_{n+1}<\alpha_{n}$. Condition (i) is needed to make sense of condition (c) of the construction, and condition (ii) is needed in the inductive proof of condition (i) for $n+1$.

The fact that $\alpha_{n+1}<\alpha_{n}$ for each $n$ is of course a contradiction. The contradiction shows that $R$ must have an infinite branch.

Let $\left\langle\delta_{n}, K_{n}, a_{n}, \pi_{n}\right| n\langle\omega\rangle$ form an infinite branch through $R$. Let $\bar{\delta}=$ $\sup \left\{\delta_{n} \mid n<\omega\right\}$ and let $\bar{A}=A^{*} \cap \mathrm{V}_{\bar{\delta}}$. Let $K_{\infty}$ be the direct limit of the chain 
$\left\langle K_{n}, \pi_{n} \mid n<\omega\right\rangle$ and let $\pi_{n, \infty}$ be the direct limit embeddings. By condition (3), $\pi_{n, \infty}\left(\delta_{n}\right)=\bar{\delta}$ and $\pi_{n, \infty}\left(A^{*} \cap \mathrm{V}_{\delta_{n}}\right)=\bar{A}$. It follows that $K_{\infty} \supset \mathrm{V}_{\bar{\delta}} \cup\{\bar{\delta}, \bar{A}\}$ and that $K_{\infty} \models \varphi[\bar{\delta}, \bar{A}]$. By condition (4) $K_{\infty}$ is illfounded. The wellfounded part of $K_{\infty}$ is admissible by standard arguments, contains $\mathrm{V}_{\bar{\delta}} \cup\{\bar{\delta}, \bar{A}\}$, and satisfies $\varphi[\bar{\delta}, \bar{A}]$ since $\varphi$ is $\Pi_{1} . \bar{\delta}$ is smaller than $\delta^{*}$ since $\delta^{*}$ has uncountable cofinality. $\quad$

The basic component of our counterexamples is an alternating chain, which we construct next. Though the precise formulations we have in this section do not appear in previous literature, the constructions are well known, tracing back to Martin-Steel [3].

REMARK 1.2. Recall that an iteration tree $\mathcal{U}$ of limit length $\theta$, consisting of a tree order $U$, and a sequence $\left\langle N_{\xi}, F_{\xi}, k_{\zeta, \xi} \mid \zeta U \xi<\theta\right\rangle$ of models, extenders, and embeddings is a plus two tree if

$$
\operatorname{strength}^{N_{\alpha}}\left(F_{\alpha}\right) \geq \sup \left\{\operatorname{crit}\left(F_{\xi}\right) \mid \xi>\alpha \wedge U-\operatorname{pred}(\xi+1) \leq \alpha\right\}+2
$$

for each $\alpha<\theta$. This technical condition is needed for the proof of iterability in Martin-Steel [3].

All the iteration trees in this paper are plus two, though we do not mention this explicitly from now on.

Definition 1.3. A length $\omega$ iteration tree consisting of a tree order $T$ and a sequence of models, extenders, and embeddings $\left\langle M_{n}, E_{n}, j_{m, n}\right| m T n\langle\omega\rangle$ is called an alternating chain just in case that for each $n \geq 2$, the $T$-predecessor of $n$ is $n-2$.

We refer to the tree order of an alternating chain as $T^{\text {alt }}$, and to the alternating chain as $\left\langle M_{n}, E_{n}, j_{m, n} \mid m T^{\text {alt }} n<\omega\right\rangle$. The tree order of an alternating chain has precisely two infinite branches: the branch $0 T^{\text {alt }} 2 T^{\text {alt }} 4 \cdots$, called even, and the branch $0 T^{\text {alt }} 1 T^{\text {alt }} 3 T^{\text {alt }} 5 \cdots$, called odd. We use $j_{n \text {,odd }}$ and $j_{n, \text { even }}$ to denote the direct limit embeddings along these branches.

Definition 1.4. For a model $Q$ and a set $A \subset Q \| \delta^{1}$ with the property that $A \cap(Q \| \gamma) \in Q$ for each $\gamma<\delta$, let us say that two embeddings $j$ and $j^{\prime}$ acting on $Q$ agree on $A$ just in case that $\bigcup_{\gamma<\delta} j(A \cap(Q \| \gamma))=\bigcup_{\gamma<\delta} j^{\prime}(A \cap(Q \| \gamma))$.

We use this terminology in cases where $j(\delta)=j^{\prime}(\delta)=\delta$. For $A$ which belong to $Q$ the statement that $j$ and $j^{\prime}$ agree on $A$ is then equivalent to the statement that $j(A)=j^{\prime}(A)$. But the former is more general, applying also when $A \notin Q$. We need the greater generality in Lemma 3.1.

Lemma 1.5. (Assuming that $\delta$ and $F$ witness conditions (A1) and (A2) above, with $\delta$ least possible.) There exists an alternating chain $\mathcal{T}=\left\langle M_{n}, E_{n}, j_{m, n}\right|$ $\left.m T^{\text {alt }} n<\omega\right\rangle$ so that:

1. $M_{0}=M_{1}=\mathrm{V}$.

2. For each $n, E_{n}$ belongs to $M_{n} \| j_{0, n}(\delta)$.

3. For each $n, \operatorname{crit}\left(E_{n}\right)>\operatorname{crit}(F)$.

4. $\operatorname{crit}\left(j_{0, \text { even }}\right)<\operatorname{crit}\left(j_{0, \text { odd }}\right)$.

5. $j_{0 \text {,even }}(\delta)=j_{0, \text { odd }}(\delta)=\delta$ (in particular both $M_{\text {even }}$ and $M_{\text {odd }}$ are wellfounded to $\delta+1)$.

\footnotetext{
${ }^{1} Q \| \delta$ in the case of a coarse model $Q$ stands for $\mathrm{V}_{\delta}^{Q}$.
} 
6. $j_{0, \text { even }}$ and $j_{0, \text { odd }}$ agree on $F$.

Proof. By Lemma 1.1, applied with $A=F$ and $\varphi(\delta, F)=" \delta$ is Woodin and $\operatorname{crit}(F)<\delta$," the minimality of $\delta$ implies that $\delta$ has cofinality $\omega$. Let $\left\langle\zeta_{n} \mid n<\omega\right\rangle$ be an increasing sequence cofinal in $\delta$. Let $K$ be the smallest admissible set containing $\mathrm{V}_{\delta} \cup\{F\}$. Andretta [1, Section 3] shows how to use the fact that $\delta$ is Woodin in $K$ to construct an alternating chain $\left\langle M_{n}, E_{n}, j_{m, n} \mid m T^{\text {alt }} n<\omega\right\rangle$ so that:

(i) $M_{0}=M_{1}=\mathrm{V}$.

(ii) For each $n, E_{n}$ belongs to $M_{n} \| j_{0, n}(\delta)$.

(iii) For each $n, \operatorname{crit}\left(E_{n+1}\right)>j_{0, n}\left(\zeta_{n}\right)$.

(iv) $\operatorname{crit}\left(j_{0, \text { even }}\right)<\operatorname{crit}\left(j_{0, \text { odd }}\right)$.

Constraining the construction there to use only extenders which are strong with respect to $F$ - there are enough such extenders for the construction as $\delta$ is Woodin in an admissible set containing $\mathrm{V}_{\delta} \cup\{F\}$ - one can obtain the additional condition:

(v) In $M_{n}, E_{n}$ is $\kappa_{n}$-strong with respect to $j_{0, n}(F)$, where $\kappa_{n}=\operatorname{crit}\left(E_{n+1}\right)$.

A simple computation using condition (iii) and the fact that $\left\langle\zeta_{n} \mid n<\omega\right\rangle$ is cofinal in $\delta$ shows that each of the sequences $\left\langle\operatorname{crit}\left(j_{n, \text { even }}\right)\right| n$ even $\rangle$ and $\left\langle\operatorname{crit}\left(j_{n, \text { odd }}\right)\right| n$ odd $\rangle$ is increasing and cofinal in $\delta$. From this it follows that both $j_{0, \text { even }}(\delta)$ and $j_{0, \text { odd }}(\delta)$ are equal to $\delta$.

Let $F_{n}=j_{0, n}(F)\left\lceil\kappa_{n}\right.$. Using condition (v) one can check that $F_{n+1}$ extends $F_{n}$ for each $n$. Let $F_{\omega}=\bigcup_{n<\omega} F_{n}$. Again using the fact that each of $\left\langle\operatorname{crit}\left(j_{n, \text { even }}\right)\right|$ $n$ even $\rangle$ and $\left\langle\operatorname{crit}\left(j_{n, \text { odd }}\right)\right| n$ odd $\rangle$ is increasing and cofinal in $\delta$ it is easy to see that $j_{0, \text { even }}(F)$ and $j_{0, \text { odd }}(F)$ are both equal to $F_{\omega}$.

REMARK 1.6. With the assumption that $\delta$ is Woodin in $K$ strengthened to the assumption that $\delta$ is outright Woodin in $\mathrm{V}$, the construction of the alternating chain in the proof of Lemma 1.5 is a well known application of the one-step lemma of Martin-Steel [2], see particularly Section 5 of that paper. Andretta $[1$, Section 3] shows how the methods of Martin-Steel [2] can be refined to apply when $\delta$ is only assumed to be Woodin in $K$. (Andretta produces iteration trees with the most general tree orders possible. The specific case of an alternating chain is due to Steel.) The reader who is familiar with the alternating chain construction of Martin-Steel [2] but not with the refinement of Andretta [1] may replace "admissible set" with "set satisfying enough of ZFC for the construction of Martin-Steel [2]" in Lemma 1.5. The replacement has no effect on the proof of the lemma and on the rest of the paper, except that the same replacement would have to be made in the hypothesis of the main results (in condition (A2) above to be precise), and the use Lemma 1.1 would be eliminated, replaced by a straightforward reflection.

REMARK 1.7. We include the last two paragraphs in the proof of Lemma 1.5 to make it clear that the proof that $j_{0, \text { even }}$ and $j_{0, \text { odd }}$ agree on $F$ uses the fact that the support of $F$ is precisely equal to $\sup _{n<\omega} \kappa_{n}$, and therefore implicitly uses the fact that $\operatorname{spt}(F)$ has cofinality $\omega$. 
$\S 2$. A counterexample to UBH. The following theorem produces a counterexample to $\mathrm{UBH}$.

TheOREm 2.1. (Assuming conditions (A1) and (A2) in Section 1.) There exists an iteration tree $\mathcal{U}$ on $\mathrm{V}$, with exactly two cofinal branches, both leading to wellfounded direct limits.

Proof. Fix an alternating chain $\mathcal{T}=\left\langle M_{n}, E_{n}, j_{m, n} \mid m T^{\text {alt }} n<\omega\right\rangle$ satisfying the conditions of Lemma 1.5. Let $M_{\text {odd }}$ and $M_{\text {even }}$ be the direct limits along the odd branch and the even branch of $\mathcal{T}$ respectively. Let $j_{\text {odd }}: \mathrm{V} \rightarrow M_{\text {odd }}$ and $j_{\text {even }}: \mathrm{V} \rightarrow M_{\text {even }}$ be the direct limit embeddings. The alternating chain is such that $j_{\text {even }}(F)=j_{\text {odd }}(F)$.

Note that since $\mathrm{V}$ and $\operatorname{Ult}(\mathrm{V}, F)$ agree to $\delta$, and since all the extenders used in $\mathcal{T}$ are taken from $\mathrm{V}_{\delta}$ and its images, $\mathcal{T}$ can be viewed as acting on $\operatorname{Ult}(\mathrm{V}, F)$. We show that the iteration tree $\mathcal{U}$ consisting of an application of $F$ to $\mathrm{V}$ followed by an application of $\mathcal{T}$ to $\operatorname{Ult}(\mathrm{V}, F)$ has both its cofinal branches wellfounded.

To be precise, let $U$ be the tree order determined by the conditions:

- $0 U 1$.

- For $n, m \geq 1, m U n$ iff $(m-1) T^{\text {alt }}(n-1)$.

Let $F_{0}=F$ and for $n \geq 1$ let $F_{n}=E_{n-1}$. Let $N_{0}=\mathrm{V}$, let $N_{1}=\operatorname{Ult}(\mathrm{V}, F)$, and let $k_{0,1}$ be the ultrapower embedding. Let $N_{2}=N_{1}$ and let $k_{1,2}$ be the identity. For $n \geq 2$ let $N_{n+1}=\operatorname{Ult}\left(N_{n-1}, F_{n}\right)$ and let $k_{n-1, n+1}$ be the ultrapower embedding. Let the remaining embeddings $k_{m, n}, m U n$, be determined by composition. Let $\mathcal{U}$ be the iteration tree $\left\langle N_{n}, F_{n}, k_{m, n} \mid m U n<\omega\right\rangle$.

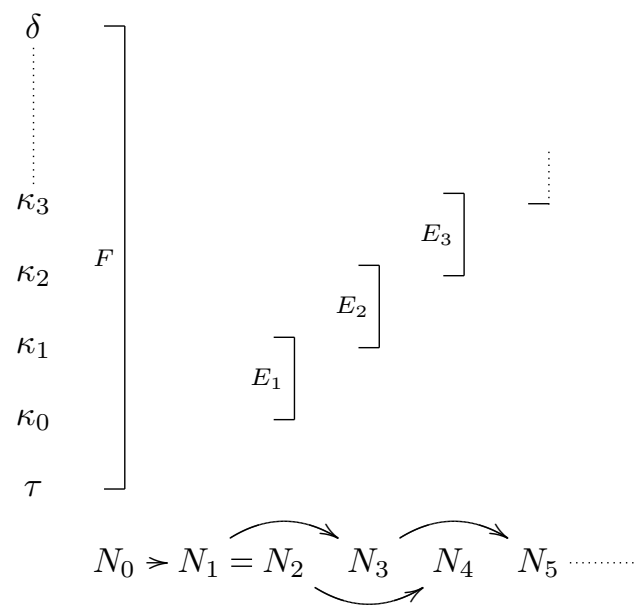

Diagram 1. The structure of $\mathcal{U}$.

$U$ has two cofinal branches, $0 U 1 U 2 U 4 U 6 \cdots$, which we refer to as the odd branch since it corresponds to the odd branch of $\mathcal{T}$, and $0 U 1 U 3 U 5 \cdots$, which we call the even branch. Let $N_{\text {odd }}$ and $N_{\text {even }}$ be the direct limits along these branches, and let $k_{n \text {,odd }}$ and $k_{n \text {,even }}$ be the direct limit embeddings. 
Since $N_{1}=\mathrm{Ult}(\mathrm{V}, F)$ and $\mathrm{V}$ have the same bounded subsets of $\delta$, the embeddings $k_{1, \text { odd }}$ and $k_{1 \text {,even }}$ agree with $j_{\text {odd }}$ and $j_{\text {even }}$ on bounded subsets of $\delta$. Since $\delta$ is a fixed point of these embeddings it follows that:

(i) $k_{1, \text { odd }}(Z) \cap \delta=j_{\text {odd }}(Z) \cap \delta$ and $k_{1 \text {,even }}(Z) \cap \delta=j_{\text {even }}(Z) \cap \delta$ for every $Z \in N_{1}$.

Recall that the support of $F$ is $\delta$. Let $\tau=\operatorname{crit}(F)$.

Claim 2.2. Every element of $N_{\text {odd }}$ has the form $k_{0, \text { odd }}(f)(a)$ for $a \in[\delta]^{<\omega}$ and $f:[\tau]^{\ln (a)} \rightarrow \mathrm{V}$.

Proof. Fix $x \in N_{\text {odd }}$. Since each of the extenders used along $k_{1 \text {,odd }}$ has critical point less than $\delta$ and support contained in $\delta$, and since $\delta$ is fixed by $k_{1, \text { odd }}, x$ can be written as $k_{1, \text { odd }}(g)(b)$ where $b \in[\delta]^{<\omega}$ and $g:[\delta]^{]^{\operatorname{h}(b)}} \rightarrow N_{1}$ with $g \in N_{1}$.

Since $g$ is an element of $N_{1}=\operatorname{Ult}(\mathrm{V}, F)$, it can be written as $k_{0,1}(h)(c)$ where $c \in[\delta]^{<\omega}$ and $h:[\tau]^{\operatorname{lh}(c)} \rightarrow \mathrm{V}$.

$x$ is therefore equal to $\left(k_{0, \text { odd }}(h)\left(k_{1, \text { odd }}(c)\right)\right)(b)$. Since $b$ and (using the fact that $\left.k_{1, \text { odd }}(\delta)=\delta\right) k_{1 \text {,odd }}(c)$ both belong to $[\delta]^{<\omega}$, and since $k_{0, \text { odd }}(\tau)>\delta$, this expression in turn can be written as $k_{0, \text { odd }}(f)(a)$, with $a \in[\delta]^{<\omega}$ and $f:[\tau]^{\ln (a)} \rightarrow$ $\mathrm{V}$.

Let $F_{\text {odd }}=j_{\text {odd }}(F)$. Since $j_{\text {odd }}(\delta)=\delta$ and $\operatorname{crit}\left(j_{\text {odd }}\right)>\tau, F_{\text {odd }}$ is an extender with support equal to $\delta$ and critical point equal to $\tau$.

Claim 2.3. Let $a \in[\delta]^{<\omega}$ and let $X \subset[\tau]^{\operatorname{lh}(a)}$. Then $X$ has $\left(F_{\text {odd }}\right)_{a}-$ measure one iff $a \in k_{0, \text { odd }}(X)$.

Proof. For an extender $E$ in a model $Q$ let $i_{E}^{Q}$ denote the ultrapower embedding of $Q$ by $E$. $X$ has $\left(F_{\text {odd }}\right)_{a}$-measure one iff $a \in i_{F_{\text {odd }}}^{\mathrm{V}}(X)$. The following equivalences lead from $a \in i_{F_{\text {odd }}}^{\mathrm{V}}(X)$ to $a \in k_{0, \text { odd }}(X)$.

$$
\begin{aligned}
a \in i_{F_{\text {odd }}}^{\mathrm{V}}(X) & \Longleftrightarrow{ }^{(1)} a \in i_{F_{\text {odd }}}^{M_{\text {odd }}}(X) \\
& \Longleftrightarrow a \in j_{\text {odd }}\left(i_{F}^{\mathrm{V}}\right)(X) \\
& \Longleftrightarrow{ }^{(2)} a \in j_{\text {odd }}\left(i_{F}^{\mathrm{V}}\right)\left(j_{\text {odd }}(X)\right) \\
& \Longleftrightarrow a \in j_{\text {odd }}\left(i_{F}^{\mathrm{V}}(X)\right) \\
& \Longleftrightarrow{ }^{(3)} a \in k_{1, \text { odd }}\left(i_{F}^{\mathrm{V}}(X)\right) \\
& \Longleftrightarrow{ }^{(4)} a \in k_{1, \text { odd }}\left(k_{0,1}(X)\right) \\
& \Longleftrightarrow a \in k_{0, \text { odd }}(X) .
\end{aligned}
$$

The equivalence marked (1) holds because $\tau$ is within the agreement between $\mathrm{V}$ and $M_{\text {odd }}$, and hence the ultrapower embeddings $i_{F_{\text {odd }}}^{\mathrm{V}}$ and $i_{F_{\text {odd }}}^{M_{\text {odd }}}$ agree on subsets of $\tau$. The equivalence marked (2) holds because $\operatorname{crit}\left(j_{\text {odd }}\right)>\tau$ so that $j_{\text {odd }}$ does not move $X$. The equivalence marked (3) holds by condition (i) above as $a \subset \delta$. The equivalence marked (4) holds because $k_{0,1}$ is the ultrapower embedding by $F$.

Claim 2.2 shows that $N_{\text {odd }}$ is isomorphic to the ultrapower of $\mathrm{V}$ by the $(\tau, \delta)$ pre-extender derived from $k_{0, \text { odd }}$. Claim 2.3 shows that this pre-extender is precisely equal to $F_{\text {odd }}$. So $N_{\text {odd }}$ is isomorphic to $\mathrm{Ult}\left(\mathrm{V}, F_{\text {odd }}\right)$. 
A similar argument applies to the even branch, demonstrating that $N_{\text {even }}$ is isomorphic to $\operatorname{Ult}\left(\mathrm{V}, F_{\text {even }}\right)$ where $F_{\text {even }}=j_{\text {even }}(F)$.

Remember now that the alternating chain $\mathcal{T}$ was obtained through an application of Lemma 1.5 , so that $j_{\text {even }}(F)=j_{\text {odd }}(F)$, i.e., $F_{\text {even }}=F_{\text {odd }}$. Thus

$$
\begin{aligned}
N_{\text {odd }} & \cong \operatorname{Ult}\left(\mathrm{V}, F_{\text {odd }}\right) \\
& =\mathrm{Ult}\left(\mathrm{V}, F_{\text {even }}\right) \\
& \cong N_{\text {even }} .
\end{aligned}
$$

By Martin-Steel [3] at least one of the infinite branches of $\mathcal{U}$ leads to a wellfounded direct limit. $(\mathcal{U}$ has precisely two infinite branches. If they both led to illfounded direct limits, the tree would be continuously illfounded.) Since the direct limit models, $N_{\text {odd }}$ and $N_{\text {even }}$, are isomorphic, they must both be wellfounded, and in fact equal.

$\square$ (Theorem 2.1)

REMARK 2.4. Our proof of Theorem 2.1 resembles the constructions of iteration trees with weaker forms of branch-ambiguity in Martin-Steel [3, 5.1,5.2].

Definition 2.5. An extender $E$ in a model $M$ is countably closed in $M$ if $\left(\operatorname{Ult}(M, E)^{\omega}\right)^{M} \subset \operatorname{Ult}(M, E)$, meaning that every countable subset of $\operatorname{Ult}(M, E)$ in $M$ belongs to $\operatorname{Ult}(M, E)$.

The following proposition shows that our method will not produce a counterexample to UBH if we demand that the first extender $F$ in our tree be countably closed.

Proposition 2.6. Let $\mathcal{T}$ be a countable iteration tree on $\mathrm{V}_{\delta}$, and let $F$ be a countably closed extender over $\mathrm{V}$ such that $\mathrm{V}_{\delta} \subset \mathrm{Ult}(\mathrm{V}, F)$. Then for any branch $b$ of $\mathcal{T}, b$ is wellfounded when $\mathcal{T}$ acts on $\operatorname{Ult}(\mathrm{V}, F)$ if and only if $b$ is wellfounded when $\mathcal{T}$ acts on $\mathrm{V}$.

Proof Sketch. Let $E$ be the long extender of the branch $b$, which is the same no matter which model we consider $\mathcal{T}$ as acting on. Let $\alpha \leq \delta$ be least such that $\mathcal{T}$ is an iteration tree on $\mathrm{V}_{\alpha}$. Suppose $\alpha$ is a limit ordinal; we leave the other case to the reader. Since $\mathcal{T}$ is countable, $\operatorname{cof}(\alpha)=\omega$, and thus $\mathrm{V}_{\alpha+1} \subset \operatorname{Ult}(\mathrm{V}, F)$. Let $M=\operatorname{Ult}(\mathrm{V}, F)$. From the fact that $M$ and $\mathrm{V}$ agree to $\alpha+1$ it follows that $i_{E}^{\mathrm{V}} \uparrow \mathrm{V}_{\alpha+1}=i_{E}^{M} \uparrow \mathrm{V}_{\alpha+1}$. Moreover, $\operatorname{Ult}(\mathrm{V}, E)$ is wellfounded iff $i_{E}^{\mathrm{V}}$ preserves wellfoundedness of relations on $\mathrm{V}_{\alpha}$, and $\operatorname{Ult}(M, E)$ is wellfounded iff $i_{E}^{M}$ preserves wellfoundedness of relations on $\mathrm{V}_{\alpha}$. So $\operatorname{Ult}(\mathrm{V}, E)$ is wellfounded iff $\operatorname{Ult}(M, E)$ is wellfounded, as desired.

An extender $F$ with strength $(F)=\operatorname{spt}(F)$ and $\operatorname{cof}(\operatorname{spt}(F))=\omega$ cannot be countably closed, since that would imply that $F \in \operatorname{Ult}(\mathrm{V}, F)$. In our particular construction, it was important for the extenders used in $\mathcal{T}$ to have critical points cofinal in the support of $F$, and consequently it was important for $\operatorname{spt}(F)$ to have cofinality $\omega$, see Remark 1.7 and the proof of Lemma 1.5. This introduced the lack of countable closure to our counterexample.

It is not known whether there is a counterexample to UBH which uses only countably closed extenders. 
§3. A counterexample to $\mathrm{CBH}$. Our counterexample to $\mathrm{CBH}$ is an iteration tree of length $\omega^{2}$, consisting of an application of the extender $F$, followed by a composition of $\omega$ shifts of the alternating chain $\mathcal{T}$. In forming the composition tree we use the even branch through each of the shifts of $\mathcal{T}$, but we use the direct limit along the odd branch to do the shifting. This mismatch, which is possible using the fact that the two branches have the same direct limit, causes the unique cofinal branch of the composition tree to be illfounded.

The following lemma will be used to shift $\mathcal{T}$ :

LEMmA 3.1. Let $\mathcal{T}=\left\langle M_{n}, E_{n}, j_{m, n}\right| m T^{\text {alt }} n\langle\omega\rangle$ be an alternating chain satisfying the conditions in Lemma 1.5. Let $\bar{\sigma}: \mathrm{V}_{\delta} \rightarrow Q \| \delta$ be elementary, where $Q$ is a model of ZFC with $\delta$ inaccessible in $Q$. Define $\bar{\sigma} \mathcal{T}$ to be the alternating chain $\mathcal{T}^{*}=\left\langle M_{n}^{*}, E_{n}^{*}, j_{m, n}^{*} \mid m T^{\text {alt }} n<\omega\right\rangle$ determined by the conditions $M_{0}^{*}=Q$ and $E_{n}^{*}=\bar{\sigma}\left(E_{n}\right)$. (These conditions determine a unique alternating chain on Q.)

Then $\mathcal{T}^{*}$ too satisfies the conditions in Lemma 1.5, with $\mathrm{V}$ replaced by $Q$ and $F$ replaced by $F^{*}=\bigcup_{\gamma<\delta} \bar{\sigma}(F\lceil\gamma)$.

Proof. This is easy to verify. Let us just note that the inaccessibility of $\delta$ in $Q$ is used to make sure that $\delta$ is a fixed point of each of the individual ultrapower embeddings used in $\bar{\sigma} \mathcal{T}$.

The following lemma abstracts one of the components of the proof of Theorem 2.1:

LEMMA 3.2. Let $\mathcal{U}$ be an iteration tree of limit length $\theta$, consisting of a tree order $U$ and a sequence $\left\langle N_{\xi}, F_{\xi}, k_{\zeta, \xi} \mid \zeta U \xi<\theta\right\rangle$ of models, extenders, and embeddings. Let $b \subset \theta$ be a cofinal branch through $\mathcal{U}$, let $N_{b}$ be the direct limit of the models along $b$, and let $k_{\xi, b}: N_{\xi} \rightarrow N_{b}$ be the direct limit embeddings. Suppose that

1. $\operatorname{spt}\left(F_{0}\right)=\operatorname{strength}\left(F_{0}\right)=\delta$.

2. The extenders $F_{\xi}$ for $\xi \geq 1$ all have supports contained in $\delta$, and critical points above $\operatorname{crit}\left(F_{0}\right)$.

3. $k_{1, b}(\delta)=\delta$.

Let $F^{*}=\bigcup_{\gamma<\delta} k_{1, b}\left(F_{0}\lceil\gamma)\right.$. Then $N_{b} \cong \operatorname{Ult}\left(\mathrm{V}, F^{*}\right)$.

PROOF. The arguments in the proof of Theorem 2.1, from Claim 2.2 onward, adapt in a straightforward way to show that $N_{b}$ and $\operatorname{Ult}\left(\mathrm{V}, F^{*}\right)$ are isomorphic.

Using this lemma, the alternating chain of Lemma 1.5, and Lemma 3.1, we now produce a counterexample to $\mathrm{CBH}$.

Definition 3.3. Let $\mathcal{U}$ be an iteration tree of length $\theta+1$, leading to a final model $N_{\theta}$. Let $\mathcal{T}$ be a length $\omega$ iteration tree on $N_{\theta}$, with a tree order $T$ and extenders $\left\langle E_{n}^{\mathcal{T}} \mid n<\omega\right\rangle$. Define $\mathcal{U} \sim \mathcal{T}$ to be the iteration tree $\mathcal{U}^{*}$ determined by the conditions: $\operatorname{lh}\left(\mathcal{U}^{*}\right)=\theta+\omega, \mathcal{U}^{*} \mid \theta+1=\mathcal{U}, E_{\theta+n}^{\mathcal{U}^{*}}=E_{n}^{\mathcal{T}}$ for each $n<\omega$, and $\theta+m U^{*} \theta+n$ iff $m T n$ for $m, n<\omega$. ( $U^{*}$ here is the tree order of $\mathcal{U}^{*}$, and $E_{\xi}^{\mathcal{U}^{*}}$ are its extenders.) $\mathcal{U}^{*}$ is the natural concatenation of $\mathcal{U}$ and $\mathcal{T}$. Its models consist of the models of $\mathcal{U}$ up to $\theta$, followed by the models of $\mathcal{T}$. 
Given a branch $b$ of $T$ define $[0, \theta]_{U} \frown b$ to be the branch $[0, \theta]_{U} \cup\{\theta+n \mid n \in b\}$ of $U^{*}$.

THEOREM 3.4. (Assuming conditions (A1) and (A2) in Section 1.) There is an iteration tree on $\mathrm{V}$, of length $\omega \cdot \omega$, with only one cofinal branch, and such that the direct limit of the models along this branch is illfounded.

Proof. Fix an alternating chain $\mathcal{T}=\left\langle M_{n}, E_{n}, j_{m, n} \mid m T^{\text {alt }} n<\omega\right\rangle$ satisfying the conditions of Lemma 1.5. Let $b_{\text {even }}$ and $b_{\text {odd }}$ denote the even branch and odd branch of $T$ respectively. Let $M_{\text {even }}$ and $M_{\text {odd }}$ be the direct limits along these branches and let $j_{\text {even }}: \mathrm{V} \rightarrow M_{\text {even }}$ and $j_{\text {odd }}: \mathrm{V} \rightarrow M_{\text {odd }}$ be the direct limit embeddings. The alternating chain is such that $j_{\text {even }}(\delta)=j_{\text {odd }}(\delta)=\delta$, and $j_{\text {even }}$ and $j_{\text {odd }}$ agree on $F$.

First we construct an auxiliary tree $\mathcal{S}$ of length $\omega^{2}$, from which the tree $\mathcal{U}$ witnessing $\neg \mathrm{CBH}$ will be obtained later. Let $\alpha_{0}=1$, and let $\alpha_{n}=\omega \cdot n$ for $n \geq 1$, so that $\alpha_{n+1}=\alpha_{n}+\omega$ for each $n$. Let $\mathcal{S}$, consisting of a tree order $S$ and a sequence $\left\langle N_{\xi}, F_{\xi}, \sigma_{\zeta, \xi} \mid \zeta S \xi<\omega \cdot \omega\right\rangle$, be the iteration tree determined by the following conditions:

1. $N_{0}=\mathrm{V}$ and $F_{0}=F$. (So that $N_{1}=\operatorname{Ult}(\mathrm{V}, F)$ and $k_{0,1}: N_{0} \rightarrow N_{1}$ is the ultrapower embedding. Notice then that $N_{1} \| \delta=\mathrm{V}_{\delta}$.)

2. $\mathcal{S}\left\lceil\alpha_{n+1}=\left(\mathcal{S}\left\lceil\alpha_{n}+1\right) \frown\left(\bar{\sigma}_{n} \mathcal{T}\right)\right.\right.$, where $\bar{\sigma}_{n}=\sigma_{1, \alpha_{n}} \uparrow \mathrm{V}_{\delta}$.

3. $\left[0, \alpha_{n+1}\right]_{S}=\left[0, \alpha_{n}\right]_{S} \frown b_{\text {odd }}$.

$\mathcal{S}$ thus involves an application of $F$, followed by $\omega$ uses of shifts of $\mathcal{T}$. Of course we have to verify that the direct limit of the models of $\mathcal{S}\left\lceil\alpha_{n+1}\right.$ along the branch $\left[0, \alpha_{n}\right]_{S} \frown b_{\text {odd }}$ is wellfounded, for otherwise we are not allowed to set $\left[0, \alpha_{n+1}\right]_{S}$ equal to this branch, as we do in condition (3).

Let us inductively assume that all the models of $\mathcal{S}\left\lceil\alpha_{n+1}\right.$ are wellfounded, and work to show that the direct limit of the models of $\mathcal{S}\left\lceil\alpha_{n+1}\right.$ along the branch $\left[0, \alpha_{n}\right]_{S} \frown b_{\text {odd }}$ is wellfounded.

Let $N_{\text {even }}^{n}$ and $N_{\text {odd }}^{n}$ be the direct limits of the models of $\mathcal{S}\left\lceil\alpha_{n+1}\right.$ along the branches $\left[0, \alpha_{n}\right]_{S} \frown b_{\text {even }}$ and $\left[0, \alpha_{n}\right]_{S} \frown b_{\text {odd }}$ respectively. Let $\sigma_{\xi \text {,even }}^{n}$ and $\sigma_{\xi \text {,odd }}^{n}$ be the direct limit embeddings.

Claim 3.5. Suppose that $\sigma_{1, \alpha_{n}}(\delta)=\delta$. Then:

- $\sigma_{\alpha_{n}, \text { even }}^{n}(\delta)=\sigma_{\alpha_{n}, \text { odd }}^{n}(\delta)=\delta$.

- $\sigma_{\alpha_{n}, \text { even }}^{n}$ and $\sigma_{\alpha_{n}, \text { odd }}^{n}$ agree on $\bigcup_{\gamma<\delta} \sigma_{1, \alpha_{n}}(F \uparrow \gamma)$.

Proof. Immediate from Lemma 3.1, applied with $Q=N_{\alpha_{n}}$ and $\bar{\sigma}=\bar{\sigma}_{n}$. Let us just note that the inaccessibility of $\delta$ in $N_{\alpha_{n}}$ follows from the assumption that $\delta=\sigma_{1, \alpha_{n}}(\delta)$ and the fact that $\delta$ is inaccessible (in fact Woodin) in $N_{1}=$ $\operatorname{Ult}(\mathrm{V}, F)$.

Notice that $\sigma_{\alpha_{n}, \alpha_{n+1}}$ is simply the map $\sigma_{\alpha_{n}, \text { odd }}^{n}$. Thus, assuming that $\sigma_{1, \alpha_{n}}(\delta)=$ $\delta$, Claim 3.5 shows that $\sigma_{1, \alpha_{n+1}}(\delta)=\delta$. By induction then we obtain:

Corollary 3.6. For each $n<\omega$ :

- $\sigma_{1, \alpha_{n}}(\delta)=\delta$.

- $\sigma_{1, \text { even }}^{n}(\delta)=\sigma_{1, \text { odd }}^{n}(\delta)=\delta$.

- $\sigma_{1, \text { even }}^{n}$ and $\sigma_{1, \text { odd }}^{n}$ agree on $F$. 
$\mathcal{S}:$

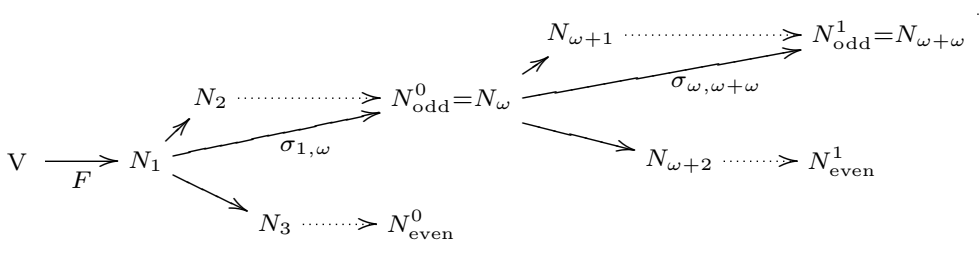

$\mathcal{U}:$

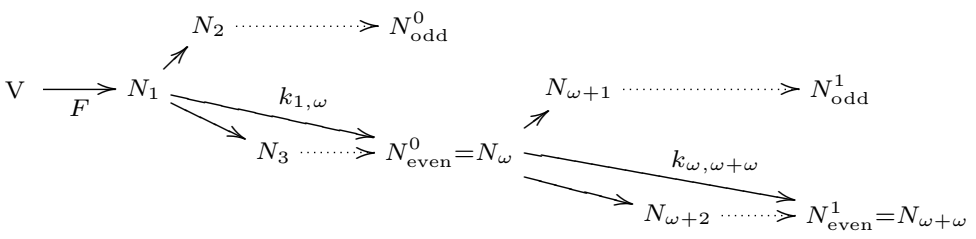

Diagram 2. The structure of $\mathcal{S}$, and the counterexample $\mathcal{U}$ to $\mathrm{CBH}$.

Claim 3.7. $N_{\text {even }}^{n}$ is isomorphic to $\operatorname{Ult}\left(\mathrm{V}, \bigcup_{\gamma<\delta} \sigma_{1, \text { even }}^{n}(F \uparrow \gamma)\right)$, and similarly $N_{\text {odd }}^{n}$ is isomorphic to $\operatorname{Ult}\left(\mathrm{V}, \bigcup_{\gamma<\delta} \sigma_{1, \text { odd }}^{n}(F\lceil\gamma))\right.$.

Proof. Immediate using Lemma 3.2 on the tree $\mathcal{S}\left\lceil\alpha_{n+1}\right.$.

Corollary 3.8. $N_{\text {even }}^{n}$ is isomorphic to $N_{\text {odd }}^{n}$.

Proof. By Corollary 3.6, $\bigcup_{\gamma<\delta} \sigma_{1 \text {,even }}^{n}\left(F\lceil\gamma)\right.$ is equal to $\bigcup_{\gamma<\delta} \sigma_{1, \text { odd }}^{n}(F\lceil\gamma)$. Using Claim 3.7 it follows that $N_{\text {even }}^{n} \cong N_{\text {odd }}^{n}$.

If both $N_{\text {even }}^{n}$ and $N_{\text {odd }}^{n}$ were illfounded, then $\bar{\sigma}_{n} \mathcal{T}$ would be a continuously illfounded iteration tree on $N_{\alpha_{n}}$, in contradiction to the results of Martin-Steel [3]. Thus at least one of $N_{\mathrm{even}}^{n}$ and $N_{\mathrm{odd}}^{n}$ is wellfounded. Since the two models are isomorphic, they must both be wellfounded. In particular $N_{\text {odd }}^{n}$, the direct limit along the branch $\left[0, \alpha_{n}\right]_{S} \smile b_{\text {odd }}$ of $\mathcal{S}\left\lceil\alpha_{n+1}\right.$, is wellfounded, so that the definition of $\mathcal{S}$, and particularly condition (3) above, is valid.

Notice that $N_{\text {odd }}^{n}$ and $N_{\text {even }}^{n}$, being transitive isomorphic models, are in fact equal.

Let $\mathcal{U}$, consisting of a tree order $U$ and a sequence $\left\langle N_{\xi}, F_{\xi}, k_{\zeta, \xi} \mid \zeta U \xi<\omega \cdot \omega\right\rangle$ of models extenders and embeddings, be the iteration tree determined by the conditions:

4. The sequence of extenders of $\mathcal{U}$ is precisely equal to the sequence of extenders of $\mathcal{S}$, and $U-\operatorname{pred}(\xi+1)=S-\operatorname{pred}(\xi+1)$ for each $\xi<\omega \cdot \omega$.

5. $\left[0, \alpha_{n+1}\right]_{U}=\left[0, \alpha_{n}\right]_{U} \frown b_{\text {even }}$ for each $n<\omega$.

The definition makes sense, and in fact $\mathcal{U}$ and $\mathcal{S}$ have precisely the same models and the same embeddings within each of the intervals $\left[\alpha_{n}, \alpha_{n+1}\right),{ }^{2}$ since $\left[0, \alpha_{n}\right]_{S} \widetilde{\smile} b_{\text {even }}$ and $\left[0, \alpha_{n}\right]_{S} \widetilde{ } b_{\text {odd }}$ both lead to the same direct limit model in $\mathcal{S}\left\lceil\alpha_{n+1}\right.$.

\footnotetext{
${ }^{2}$ By this we mean that $k_{\zeta, \xi}=\sigma_{\zeta, \xi}$ whenever $\zeta$ and $\xi$ belong to the same interval $\left[\alpha_{n}, \alpha_{n+1}\right)$.
} 
Of course, the embeddings $k_{\alpha_{n}, \alpha_{n+1}}$ and $\sigma_{\alpha_{n}, \alpha_{n+1}}$ are not the same. The former is taken along the even branch of $\bar{\sigma}_{n} \mathcal{T}$, while the latter is taken along the odd branch.

Let $\kappa_{\text {even }}$ be the critical point of $j_{0 \text {,even }}^{\mathcal{T}}$, and let $\kappa_{\text {odd }}$ be the critical point of $j_{0 \text {,odd }}^{\mathcal{T}}$. More generally, let $\kappa_{\text {even }}^{n}$ be the critical point of the direct limit embedding along the even branch of $\bar{\sigma}_{n} \mathcal{T}$, and define $\kappa_{\text {odd }}^{n}$ similarly with the odd branch.

Notice then that:

(i) $\operatorname{crit}\left(\sigma_{\alpha_{n}, \alpha_{n+1}}\right)=\kappa_{\text {odd }}^{n}$.

(ii) $\operatorname{crit}\left(k_{\alpha_{n}, \alpha_{n+1}}\right)=\kappa_{\text {even }}^{n}$

Now $\kappa_{\text {even }}^{n}$ is simply $\sigma_{1, \alpha_{n}}\left(\kappa_{\text {even }}\right)$, and similarly $\kappa_{\text {odd }}^{n}$ is simply $\sigma_{1, \alpha_{n}}\left(\kappa_{\text {odd }}\right)$. By Claim 1.5, $\kappa_{\text {even }}<\kappa_{\text {odd }}$. Certainly then $\kappa_{\text {even }}<\sigma_{1, \alpha_{n}}\left(\kappa_{\text {odd }}\right)$ for each $n$. By condition (i), $\sigma_{1, \alpha_{n}}\left(\kappa_{\text {odd }}\right)=\kappa_{\text {odd }}^{n}$ is the critical point of $\sigma_{\alpha_{n}, \alpha_{n+1}}$. So $\kappa_{\text {even }}$ is not moved by $\sigma_{\alpha_{n}, \alpha_{n+1}}$. By induction on $n$ then it follows that $\sigma_{1, \alpha_{n}}\left(\kappa_{\text {even }}\right)=\kappa_{\text {even }}$. In other words:

(iii) $\kappa_{\text {even }}^{n}=\kappa_{\text {even }}$.

But now by condition (ii),

(iv) $k_{\alpha_{n}, \alpha_{n+1}}\left(\kappa_{\text {even }}\right)>\kappa_{\text {even }}$ for each $n<\omega$.

The iteration tree $\mathcal{U}$ has precisely one cofinal branch, the branch generated by the set $\left\{\alpha_{n} \mid n<\omega\right\}$. Let $N_{\infty}$ be the direct limit along this branch, and let $k_{\alpha_{n}, \infty}: N_{\alpha_{n}} \rightarrow N_{\infty}$ be the direct limit embeddings. Condition (iv) shows $N_{\infty}$ is illfounded: $\left\langle k_{\alpha_{n}, \infty}\left(\kappa_{\text {even }}\right) \mid n<\omega\right\rangle$ forms an infinite descending sequence of ordinals of $N_{\infty}$.

$\square$ (Theorem 3.4)

\title{
REFERENCES
}

[1] Alessandro Andretta, Large cardinals and iteration trees of height $\omega$, Ann. Pure Appl. Logic, vol. 54 (1991), no. 1, pp. 1-15.

[2] Donald A. Martin and John R. Steel, A proof of projective determinacy, J. Amer. Math. Soc., vol. 2 (1989), no. 1, pp. 71-125.

[3] - Iteration trees, J. Amer. Math. Soc., vol. 7 (1994), no. 1, pp. 1-73.

[4] Itay Neeman, Inner models in the region of a Woodin limit of Woodin cardinals, Ann. Pure Appl. Logic, vol. 116 (2002), no. 1-3, pp. 67-155.

[5] - The determinacy of long games, de Gruyter Series in Logic and its Applications, vol. 7, Walter de Gruyter GmbH \& Co. KG, Berlin, 2004.

[6] John R. SteEL, Local $K^{c}$-constructions, To appear.

[7] - Core models with more Woodin cardinals, J. Symbolic Logic, vol. 67 (2002), no. 3, pp. 1197-1226.

\author{
DEPARTMENT OF MATHEMATICS \\ UNIVERSITY OF CALIFORNIA LOS ANGELES \\ LOS ANGELES, CA 90095-1555 \\ E-mail: ineeman@math.ucla.edu \\ DEPARTMENT OF MATHEMATICS \\ UNIVERSITY OF CALIFORNIA BERKELEY \\ BERKELEY, CA 94720-3840 \\ E-mail: steel@math.berkeley.edu
}

